Sensory and autonomic nervous system examination was normal. Deep tendon reflexes were absent. Plantars were bilaterally down going.

Investigations showed normal hemogram and metabolic parameters. Creatine phosphokinase (CPK) level was normal. Human immunodeficiency virus (HIV) and hepatic viral markers were negative. Urine was negative for porphobilinogen. Cerebrospinal fluid (CSF) examination showed 4 cells $/ \mu \mathrm{L}$ (all lymphocytes), protein $76 \mathrm{mg} / \mathrm{dl}$ and sugar $40 \mathrm{mg} / \mathrm{dl}$ (corresponding blood sugar $90 \mathrm{mg} / \mathrm{dl}$ ). Electrophysiological examination of median nerves from position 1-wrist and position 2-elbow revealed decreased compound muscle action potential (CMAP) amplitude (2.1 mA and $1.2 \mathrm{~mA})$, with reduced conduction velocity $18.6 \mathrm{~m} / \mathrm{s}$ and conduction block (more than $40 \%$ decrement in CMAP amplitude). ' $F$ ' waves were not generated in any of the limbs. Sensory nerve conduction studies showed normal latency amplitude and conduction velocity. Repetitive nerve stimulation study did not show decremental pattern. Electromyography study done in the second week showed spontaneous

\title{
Acute polyradiculoneuropathy following wasp sting
}

Sir,

A variety of unusual systemic manifestations have been described following the exposure to Hymenoptera venom. The venom contains phospholipase- $\mathrm{A}_{2}$, hyaluronidase, mast cell degranulation protein, apamin and adolapine. ${ }^{[1]}$ Neurological complications are rare and include encephalitis, encephaloradiculoneuropathy, optic neuropathy ${ }^{[1]}$ and neuromuscular paralysis. ${ }^{[2]}$ We report a young man who developed acute radiculoneuropathy following a wasp sting.

A 20-year-old, non-hypertensive healthy man who had previous history of wasp sting without systemic complications on three earlier episodes presented with acute and rapidly progressive quadriparesis with truncal weakness after fourth episode of wasp sting. There was mild local swelling over right cheek and right arm. There was no history of tingling, paresthesia, visual blurring, diplopia, dysphasia, vomiting, convulsion, altered sensorium, syncope, bowel or bladder incontinence, oliguria, dyspnoea, palpitation, fever, drug intake or systemic diseases. Neurological examination revealed a conscious and well oriented male with normal cranial nerves' examination. Motor examination showed normal bulk, decreased tone in all four limbs and power of Grade 3/5 proximally and 4/5 distally in the limbs. Truncal weakness was present. activity in the form of fasciculations with long-duration polyphasic motor unit potentials, late recruitment pattern and incomplete interference pattern. Electrophysiological study conclusion was pure motor demyelinating radiculoneuropathy with secondary axonal changes. Sural nerve biopsy showed segmental demyelination. Patient was managed with high-dose intravenous methylprednisolone pulse therapy for five days followed by tapering dose of oral steroid, and anti-allergic treatment. The patient showed partial improvement in symptoms. He was discharged on tapering dose of oral prednisolone and supportive physiotherapy.

Hymenoptera sting's complications range from local to severe systemic reactions and even death. Reactions usually begin within a minute to an hour in $76-96 \%$ of patients. Nevertheless, there are reports of delayed responses that can occur days to weeks after the event. Out of 2606 reactions noted in a survey, complication did not occur until several days after the sting in 2.8\% cases. ${ }^{[3]}$ Various reported neurological complications include: Myelitis, encephalitis, optic neuropathy, encephaloradiculoneuritis, ${ }^{[1]}$ cerebral infarction, ${ }^{[4]}$ and acute inflammatory polyradiculopathy. ${ }^{[5]}$ Maltzman et al., reported two patients with optic neuropathy following wasp stings. Most of the patients recovered with corticosterioid..$^{[1]}$ These reactions to wasp venom are either Type I or delayed type of hypersensitivity reactions. Bachman et al., reported five patients with acute inflammatory polyradiculopathy following hymenoptera sting, in all the patients good clinical recovery was achieved with steroid. ${ }^{[5]}$ Although exact 
pathogenesis is not known it is postulated that the venom contains allergic proteins like phospholipase and various non-myelin proteins which elicit an $\operatorname{IgE}$ antibody response resulting in mast cell activation and release of preformed substances such as histamine as well as de novo synthesis of other mediators (i.e. thromboxane and leukotrines) leading to anaphylactic response. Sometimes IgE antibody formed by previous sensitization directly cross-reacts with myelin basic proteins resulting in different neurological complications. ${ }^{[6]}$

Our patient was pre-sensitized with hymenoptera venom and the fourth occasion of sting probably caused immediate type hypersensitivity reaction mediated by humoral antibodies of IgE type or reagin antibodies which cross-reacted with myelin basic protein, leading to acute demyelinating polyradiculoneuropathy with secondary axonal changes.

\section{Dhiraj Kishore, Vishal Khurana, Indarjeet Singh Gambhir, Surender Misra ${ }^{1}$}

Department of Internal Medicine and ${ }^{1}$ Neurology, Institute of Medical Sciences, Banaras Hindu University, Varanasi - 221 005, India. E-mail: vishdoc24@gmail.com

PMID: 19934578

DOI: $10.4103 / 0028-3886.57796$

\section{References}

1. Maltzman JS, Lee AG, Miller NR. Optic neuropathy occurring after bee and wasp sting. Ophthalmology 2000; 107:193-5.

2. Hira HS, Mittal A, Kumar SA, Singh A, Ahalawat RS. Myasthenia gravis and acute respiratory muscle paralysis following wasp sting. Indian J Chest Dis Allied Sci 2005;47:197.

3. Barr SE. Allergy to Hymenoptera stings-review of the world literature: 1953-1970. Ann Allergy 1971; 29:49-66.

4. Crawley F, Schon F, Brown MM. Cerebral infarction: A rare complication of wasp sting. J Neurol Neurosurg Psychiatry 1999; 66:550-1.

5. Bachman DS, Paulson GW, Mandell JR. Acute inflammatory polyradiculoneuropathy following Hymnoptera sting. JAMA 1982; 247:1443-5.

6. Mean ED, Barron KD, Van Dyne BJ. Nervous system lesions after sting by yellow jacket. A case report. Neurology 1973; 23:881-9.

Accepted on 06-08-2009 